\title{
Der Charakter eines Vertrages zwischen Veranstalter und Solist als Dienstvertrag
}

Amtsgericht Münster, Urteil vom 07. März 2008 - 60 C 4365/07

\section{Der Vertrag eines Veranstalters mit einem Solisten, der im Rahmen eines Konzerts (hier: Oratorium) zu sin- gen hat, ist Dienstvertrag.}

2. In diesem Rahmen erbringen Sänger keine „Dienste höherer Art“ (\$ 627 I BGB). (Leitsätze des Einsenders*)

\section{Tatbestand}

- Die Parteien streiten um ein Ausfallhonorar eines Künstlers.

Der Kläger ist freiberuflicher Konzertsänger. Der Beklagte ist Kirchenmusiker und u. a. als Chorleiter und Dirigent tätig. Mitte Januar haben die Parteien ein Telefongespräch geführt und darüber gesprochen, dass der Kläger bei einer für den 09. September 2007 vorgesehenen Aufführung des Oratoriums "Elias" von Felix Mendelssohn-Bartholdy in der Mutterhauskirche der Franziskanerinnen in Münster nebst einer vorangegangenen Probe am Samstag, den 08. September 2007, die Tenor-Solo-Partien aus diesem Werk singen soll. Die Vergütung sollte $800,00 €$ inclusive aller Fahrtkosten betragen.

Mit E-Mail vom 20. Januar 2007 bestätigte der Beklagte die "telefonisch erörterten Vereinbarungen“. Wegen der Einzelheiten dieser E-Mail wird auf die Anlage K 1 [...] Bezug genommen. Danach hat es keine weiteren Gespräche mehr zwischen den Parteien gegeben. Erst mit E-Mail vom 13. März 2007 teilte der Beklagte dem Kläger mit, dass das Konzert an dem zuvor besprochenen Wochenende im September nicht stattfinden könne, weil das Orchester abgesagt habe. Er müsse daher leider den Termin verlegen. Als Ausweichtermin bot der Kläger dem Beklagten den 15. und 16. September an.

Mit E-Mail vom 14. März 2007 teilte der Kläger dem Beklagten mit, dass er an dem neuen Termin vom 15. und 16. September 2007 nicht mitwirken könne, weil er schon anderweitig verpflichtet sei. Er bat den Beklagten, ihn zurückzurufen, um ein Ausfallhonorar zu verhandeln. Da sich der Beklagte nicht wieder meldete, wandte sich der Kläger mit E-Mail vom 11. April 2007 erneut an den Beklagten und forderte sein Ausfallhonorar ein. Er bot an, statt des tatsächlichen Ausfalls in Höhe von 800,00€ lediglich 500,00 € geltend zu machen, wenn dieses Ausfallhonorar bis zum 25. April 2007 auf seinem Konto gut geschrieben worden sei. Er kündigte an, dass im Fall Nichtzahlung Rechtsanwälte eingeschaltet werden, um die gesamte Forderung in Höhe von $800,00 €$ einzufordern.

Mit E-Mail vom 03. Mai 2007 äußerte der Beklagte die Rechtsauffassung, dass ihm ein Festhalten an den Vertrag, wenn er denn überhaupt zustande gekommen sei, wegen des Ausfalls

Eingesendet von Dr. Rochus Schmitz, siehe seine Urteilsanmerkung in diesem Heft, S. $55 \mathrm{f}$. des Orchesters, den er nicht zu vertreten habe, nicht zumutbar sei. Er erklärte den Rücktritt von dem Vertrag. Sodann meldete sich der Anwalt des Klägers mit Schreiben vom 10. Mai 2007 und forderte den Beklagten erneut auf, die Ausfallentschädigung in Höhe von $800,00 €$ zuzüglich Rechtsanwaltskosten bis zum 30 . Mai 2007 zu zahlen.

Der Kläger behauptet, es sei bereits bei dem ersten Telefongespräch Mitte Januar 2007 eine verbindliche Vereinbarung zustande gekommen, welche der Beklagte dann durch E-Mail vom 20. Juli 2007 bestätigt habe.

Der Kläger beantragt,

den Beklagten zu verurteilen, an ihn 782,40€ nebst $5 \%$ Zinsen über dem Basiszinssatz seit dem 26. April 2007 sowie 120,67 $€$ vorgerichtliche Anwaltskosten zu zahlen.

Der Beklagte beantragt,

die Klage abzuweisen.

Der Beklagte trägt vor, in dem Telefongespräch von Mitte Januar 2007 sei über sämtliche Vertragsbestandteile, wie Datum der Proben und der Aufführung, Aufführungsstück und Honorar bereits gesprochen worden. Er habe die E-Mail vom 20. Januar 2007 abgeschickt, um sich selbst abzusichern. In der Branche zähle das Wort und er habe deswegen auch nicht noch weiter nachgefragt, obwohl der Kläger eine weitere Bestätigung auf die E-Mail vom 20. Januar 2007 nicht übersandt habe. In der Branche müsste man allerdings eine gewisse Flexibilität besitzen, die dazu führe, dass auch mal kurzfristig Konzerte ausfallen.

Der Beklagte vertritt die Auffassung, dass ein Dienstvertrag vorliege. Dieser Dienstvertrag sei sowohl durch inn mit Mail vom 03. Mai 2007 als auch später durch seinen Prozessbevollmächtigten wirksam gekündigt worden. Deswegen könne sich auch aus dem Dienstvertrag kein Anspruch auf eine Ausfallentschädigung ergeben. (Tatbestand von der Redaktion bearbeitet.)

\section{Entscheidungsgründe}

Die Klage ist begründet.

Der Kläger hat gegen den Beklagten einen Anspruch auf Zahlung einer Ausfallentschädigung aus § 615 BGB. 
In Literatur und Rechtsprechung ist streitig, ob es sich bei dem Vertrag eines Veranstalters mit einem Sänger, der im Rahmen einer Vorführung eine Gesangseinlage darzubieten hat, um einen Dienst- oder einen Werkvertrag handelt. Das Gericht vertritt insoweit die Auffassung, dass es sich bei Verträgen mit Künstlern, die auf Mitwirkung an der Aufführung gerichtet sind, um Dienstverträge handelt. Dies gilt auch bezogen auf den streitgegenständlichen Vertrag zwischen den Parteien. Die Darbietung eines Sologesangs führt zu keinem bestimmten Erfolg bzw. Werk, sondern stellt die Darbietung einer bloßen Dienstleistung dar.

Das Gericht ist ferner davon überzeugt, dass ein verbindlicher Vertrag zwischen den Parteien zustande gekommen ist.

Nach der persönlichen Anhörung des Beklagten im Termin ist bereits fraglich, ob der Beklagte dies überhaupt noch bestreiten will. Er hat sich dahingehend eingelassen, dass in dem Telefongespräch Mitte Januar 2007 zwischen den Parteien sämtliche Bestandteile eines entsprechenden Vertrages besprochen worden sind, und dass man insoweit auch Einigung erzielt habe. So wurde der Tag der Proben sowie der Tag der Veranstaltung, die zu singende Tenor-Solo-Partie und auch die Höhe der Vergütung festgelegt. Der Beklagte hat sich darüber hinaus weiter dahingehend eingelassen, dass er die E-Mail vom 20. Januar 2007 übersandt habe, um die Vereinbarung wirklich zu manifestieren, und eine schriftliche Sicherheit zu haben. In der Branche zähle noch das Wort. Er habe aber um eine Bestätigung gebeten, welche seitens des Beklagten nicht erfolgt sei. Auf Vorhalt des Gerichts, wieso er denn Wochen lang nichts unternommen habe, obwohl eine Bestätigung des Klägers nicht mehr erfolgt sei, ob er nicht vielleicht gedacht habe, dass mit seiner Bestätigungs-Mail vom 20. Januar 2007 alles in Ordnung gewesen sei, hat der Beklagte mit "jein“ geantwortet. Einerseits sei für ihn alles klar gewesen, andererseits sei in dieser Branche eine gewisse Flexibilität von Nöten, die es dem Veranstalter erlaube, auch kurzfristig Termine abzusagen.

Selbst wenn man die Einlassung des Beklagten als Bestreiten einer verbindlichen Vereinbarung auslegen sollte, ist das Gericht unter Zugrundelegung der Aussagen beider Parteien und der entsprechenden E-Mails, welche zwischen den Parteien gewechselt worden sind, davon überzeugt, dass die Parteien bereits eine verbindliche Vereinbarung am Telefon abgeschlossen haben. Unstreitig war in dem Telefongespräch eine Einigung bezogen auf sämtliche wesentliche Vertragsbestandteile, wie oben dargestellt, erfolgt. Der Beklagte hat sogar erklärt, dass er sich im Rahmen dieses Gesprächs bereit erklärt habe, auf ein Erscheinen des Klägers zur Probe zu verzichten, wenn dieser für diesen Tag noch eine andere Verpflichtung habe.

Auch der Wortlaut der Bestätigungs-Mail des Beklagten, wonach er die "telefonisch erörterte Vereinbarung" bestätigt, spricht dafür, dass der Beklagte von einer festen Vereinbarung ausgegangen ist. Der Einleitungssatz der Mail lautet ferner, dass er sich freue, dass erneut ein gemeinsames Musizieren zustande komme. Auch dies spricht dafür, dass der Beklagte genauso wie der Kläger fest davon ausgegangen ist, dass eine entsprechende Vereinbarung bereits existiert. Auch hat der Beklagte selbst erklärt, dass die Mail zur Sicherheit versandt worden sei, damit etwas Schriftliches vorhanden sei.

Das Gericht misst der Tatsache, dass der Beklagte seine Mail mit dem Satz beendet: „Bitte um Bestätigung!“ keine besondere Bedeutung zu. Wenn der Beklagte tatsächlich davon ausgegangen sein sollte, dass es einer Bestätigung des Klägers für eine verbindliche Vereinbarung noch bedurft hätte, hätte nichts näher gelegen, als in den Wochen danach diese Bestätigung noch einmal anzumahnen. Dies hat der Beklagte aber gerade nicht getan. Dies zeigt nach der Auffassung des Gerichts eindeutig, dass auch der Beklagte von einer verbindlichen Vereinbarung bezogen auf die Teilnahme des Klägers an dem Konzert ausgegangen ist.

Der Dienstvertrag ist durch den Beklagten auch nicht wirksam gekündigt worden.

Eine ordentliche Kündigung gemäß § 621 Ziffer 5 BGB scheitert bereits daran, dass es sich bei der hier maßgeblichen Verpflichtung des Beklagten nur um eine einmalig zu erbringende Dienstleistung handelt, so dass die Voraussetzungen des $§ 620$ Absatz 2 BGB nicht vorliegen, die zu einer Kündigungsmöglichkeit nach § 621 BGB führen würden. Die Regelung des § 621 BGB ist nur auf Dienstverhältnisse anwendbar, deren Dauer aus der Beschaffenheit und dem Zweck der Dienste nicht entnommen werden kann (Ermann/Belling § 621 Rn. 1).

Es liegt auch kein Kündigungsgrund iSv § 627 BGB vor. Der Beklagte hat weder Dienste höherer Art zu leisten gehabt, noch war ihm der Dienst aufgrund besonderen Vertrauens übertragen worden. Diese Kündigungsvorschrift hat bestimmte freie Berufe im Blick, nicht jedoch einmalig ausgeübte Gesangsdarbietungen (vgl. mit Beispielen Ermann aaO, Rn. 3). Auch sind keine Anhaltspunkte für ein besonderes Vertrauensverhältnis ersichtlich. Damit scheidet die Anwendbarkeit von § 627 BGB aus.

Der Beklagte hat ferner kein Kündigungsrecht aus § 626 BGB. Dieser Kündigungsgrund greift nur dann durch, wenn Tatsachen vorliegen, aufgrund derer dem Kündigenden unter Berücksichtigung aller Umstände des Einzelfalls und in Abwägung der Interessen beider Vertragsteile die Fortsetzung des Dienstverhältnisses bis zum Ablauf der Kündigungsfrist oder bis zur vereinbarten Beendigung des Dienstverhältnisses nicht zugemutet werden kann.

Die Voraussetzungen dieser Norm liegen schon deswegen nicht vor, weil die Abwägung der Interessen beider Vertragsparteien ergibt, dass das Risiko des unerwarteten Ausfalls des Orchesters allein der Veranstalter, d. h. hier der Kläger zu tragen hat. Nur dem Kläger war es möglich, das Risiko vor Beginn der Tournee abzuschätzen. Dem Kläger standen insoweit keine Er- 
kenntnismöglichkeiten zur Verfügung. Die Störung wurde nicht von einer Partei, sondern durch einen Dritten verursacht, mit dem nur der Beklagte, nicht dagegen der Kläger, in einem Vertragsverhältnis stand. Das Risiko, dass der Vertragspartner des Beklagten sich nicht vertragsgerecht verhält, kann er nicht auf den Kläger überwälzen (vgl. dazu BGH, NJW 2002, 595).

Der Beklagte befand sich auch mit der Annahme des Dienstes des Klägers im Annahmeverzug gemäß § 615 BGB. Der Beklagte hat durch seine Mail vom 13. März 2007 zum Ausdruck gebracht, dass er die Dienstleistung des Klägers für das vereinbarte Wochenende vom 8. und 9. September nicht mehr annehmen wolle und könne. Für den Annahmeverzug ist es unter diesen Umständen gleichgültig, ob der Dienstherr die Dienstleistung nicht annehmen will oder ob er sie wegen äußerer Umstände nicht annehmen kann (Palandt-Weidenkaff $\S 615, \mathrm{Rn} .8 / 9$ ). Aus diesen Gründen brauchte der Kläger seine Dienstleistung an dem vereinbarten Tag auch nicht erneut anzubieten, weil nach den Gesamtumständen klar war, dass der Beklagte diese Dienstleistung nicht mehr annehmen würde. Nach ständiger Rechtsprechung ist darüber hinaus das Angebot der Dienstleistung auch stets dann überflüssig, wenn eine unwirksame Kündigung vorliegt. Dies war hier ebenfalls gegeben, weil der "Rücktritt vom Vertrag" des Beklagten mit Mail vom 03. Mai 2007 vorgelegen hat. Mithin hat der Beklagte sich im Annahmeverzug mit der Dienstleistung befunden.

Soweit die Parteien zunächst über die ersparten Aufwendungen gestritten haben, hat der Beklagte mit Schriftsatz vom 25. Februar unstreitig gestellt, dass der Beklagte kein Fahrzeug besessen oder benutzt hat, und dass er am 16. September 2007 andernorts engagiert war, und deswegen den Ausweichtermin nicht angenommen hat. Daher brauchten auch die schriftlich eingeholten Zeugenaussagen nicht mehr in den Prozess eingeführt werden.

Der Kläger hat gegen den Beklagten ferner einen Anspruch auf Zahlung der geltend gemachten Zinsen, §§ 280 Absatz 1, Absatz 2 iVm § 286 Absatz 1, 288 Absatz 1 BGB.

Mit E-Mail vom 11. April 2007 hat der Kläger dem Beklagten eine Frist zur Zahlung des Ausfallhonorars bis zum 25. April 2007 gesetzt und erklärt, dass er danach anwaltlich nicht nur einen geminderten Betrag von $500,00 €$, sondern von $800,00 €$ einfordern werde. Der Beklagte befand sich damit seit dem 26. April 2007 mit der Zahlung der gesamten Ausfallentschädigung in Höhe von $800,00 €$ in Verzug.

Der Kläger hat gegen den Beklagten ferner einen Anspruch auf Zahlung der Anwaltsvergütung aus $\S 280$ Absatz 1, Absatz 2 iVm § 286 Absatz 1 BGB.

Wie bereits in der Mail vom 11. April 2007 angekündigt, hat sich der Kläger, nachdem er den Beklagten wirksam in Verzug gesetzt hat, einen Anwalt gesucht, der dann den Beklagten er- neut aufgefordert hat, die Ausfallentschädigung, sowie seine Anwaltsvergütung zu zahlen. Damit waren die entstandenen Anwaltskosten Folge des Zahlungsverzuges des Beklagten.

Die Nebenentscheidungen folgen aus $\S \S 91$ Absatz 1, $708 \mathrm{Nr}$. 11, 711 ZPO. (Entscheidungsgründe von der Redaktion bearbeitet.) 